\title{
Compostos fenólicos e capacidade antioxidante de extratos de flor de Clitoria ternatea
}

\author{
L. \\ Phenolic compounds and antioxidant capacity of the Clitoria ternatea L. flower extracts \\ Compuestos fenólicos y capacidade antioxidante de extractos de flores de Clitoria ternatea $\mathrm{L}$.
}

João Vítor do Nascimento

ORCID: https://orcid.org/0000-0003-4931-6551 Universidade Tecnológica Federal do Paraná, Brasil E-mail: joaonascimento.2020@alunos.utfpr.edu.br

Vanessa Cipriani Giuliangeli

ORCID: https://orcid.org/0000-0001-6696-9701 Universidade Tecnológica Federal do Paraná, Brasil E-mail: vacipriani@gmail.com

Talita Kato

ORCID: https://orcid.org/0000-0002-0622-4844 Universidade Tecnológica Federal do Paraná, Brasil

E-mail: talita_kato@ hotmail.com

Caroline Maria Calliari

ORCID: https://orcid.org/0000-0002-2448-5546 Universidade Tecnológica Federal do Paraná, Brasil E-mail: calliari@utfpr.edu.br

Marianne Ayumi Shirai

ORCID: https://orcid.org/0000-0003-0795-6358 Universidade Tecnológica Federal do Paraná, Brasil E-mail: marianneshirai@utfpr.edu.br

\begin{abstract}
Resumo
A Clitoria ternatea L. (CT) é uma planta nativa da Ásia Tropical, popularmente identificada no Brasil como feijãoborboleta. Seus extratos possuem uma ampla gama de atividades farmacológicas, além disso as flores da CT possuem significativa quantidade de compostos fenólicos e antocianinas. O objetivo geral deste estudo foi avaliar o efeito da concentração de etanol na extração de compostos fenólicos da flor de CT e determinar a capacidade antioxidante in vitro por intermédio da metodologia de redução do ferro (FRAP) e pelo método de eliminação do radical livre 2,2difenil-1-picril-hidrazil (DPPH). Os resultados apresentados pela pesquisa evidenciaram que a água em temperatura ambiente mostrou-se como melhor solvente para extração de compostos fenólicos das flores de CT. Entre os métodos de atividade antioxidante avaliados, apenas o FRAP mostrou forte correlação com a concentração de compostos fenólicos totais. Os resultados deste estudo demonstram extrema relevância devido a capacidade de extração de bioativos que podem ser empregados na indústria alimentícia.
\end{abstract}

Palavras-chave: Composto bioativo; DPPH; FRAP; Feijão borboleta.

\begin{abstract}
Clitoria ternatea L. (CT) is a plant native from Tropical Asia, popularly identified in Brazil as butterfly bean. Its extracts have a wide range of pharmacological activities, in addition, CT flowers have a significant amount of phenolic compounds and anthocyanins. The general goal of this study was to evaluate the effect of ethanol concentration on the extraction of phenolic compounds from CT flower and also evaluate the antioxidant capacity by the method based on the elimination of the free radical 1,1-diphenyl-2-picrylhydrazy (DPPH) and iron reduction (FRAP). The results presented by the research demonstrated that water at room temperature proved to be the best solvent for extracting phenolic compounds from CT flowers. Among the evaluated methods of antioxidant activity, only FRAP showed a strong correlation with the concentration of total phenolic compounds. The results of this study are extremely relevant due to the capacity of bioactive extraction that could be employed in the food industry.

Keywords: Bioactive compound; DPPH; FRAP; Butterfly beans.

\section{Resumen}

Clitoria ternatea L. (CT) es una planta originaria de Asia tropical, popularmente identificada en Brasil como frijol mariposa. Sus extractos tienen una amplia gama de actividades farmacológicas, además, las flores de CT tienen una cantidad significativa de compuestos fenólicos y antocianinas. El objetivo general de este estudio fue evaluar el efecto de la concentración de etanol en la extracción de compuestos fenólicos de la flor de CT y también evaluar la
\end{abstract}


capacidad antioxidante por el método basado en la eliminación del radical libre 1,1-difenil-2-picrylhydrazy (DPPH) y reducción de hierro (FRAP). Los resultados presentados por la investigación demostraron que el agua a temperatura ambiente resultó ser el mejor solvente para extraer compuestos fenólicos de flores CT. Entre los métodos evaluados de actividad antioxidante, solo FRAP mostró una fuerte correlación con la concentración de compuestos fenólicos totales. Los resultados de este estudio son sumamente relevantes debido a la capacidad de extracción bioactiva que podría emplearse en la industria alimentaria.

Palabras clave: Compuesto bioactivo; DPPH; FRAP; Frijoles mariposa.

\section{Introdução}

A biodiversidade brasileira é mundialmente conhecida por ser abundante, no entanto, sendo o Brasil um país agrário, de monocultura e que foca em cultivos específicos, o aproveitamento de espécies nativas e com diversos potenciais são em sua grande maioria deixados de lado. Nesse quadro, surgem as Plantas Alimentícias não Convencionais (PANC), que crescem em diferentes regiões, geralmente de forma espontânea, e que recentemente está sendo alvo de estudos pela liga acadêmica por possuírem efeitos antioxidantes e antimicrobianos, além dos seus diversos valores nutricionais (Oliveira, 2019). Tais efeitos se devem às atividades biológicas de seus constituintes como os compostos fenólicos, vitaminas, carotenoides e minerais (Oliveira \& Anunciação, 2007).

Dentre a diversidade de PANC, tem-se a Clitoria ternatea L. (CT), originária da Ásia tropical e mais tarde disseminada na América do Sul, a CT é popularmente identificada como feijão-borboleta, cunhã, ervilha-borboleta, ismênia e palheteira. Pertence à família Fabaceae e a planta possui flores em formato de concha e com coloração azul e branca. As flores da CT possuem significativa quantidade de compostos fenólicos e antocianinas (Zingare \& Zingare, 2013) e são consumidas na forma de chá, utilizadas como corantes e no enriquecimento nutricional de diversos alimentos e bebidas.

Os compostos fenólicos são produtos secundários do metabolismo vegetal e possuem em sua estrutura química hidroxilas e anéis aromáticos, responsáveis pela capacidade antioxidante. Entre os compostos fenólicos, tem-se o grupo dos flavonoides que são divididos em subgrupos no qual se encontram as antocianinas, que são caracterizadas como pigmentos solúveis em água, com variação de cor entre o vermelho ao violeta e azul. Estruturalmente são constituídas por glicosídeos como D-glicose, D-ramnose e D-galactose (Castañeda-Ovando \& Pacheco-Hernández, 2009; Boroski et al., 2015; Aguiar, 2017). A pigmentação das antocianinas varia, dependendo do grau de hidroxilação, padrão de glicosilação, padrão de metilação dos anéis aromáticos e do pH (Arnoso et al., 2019). As antocianinas são compostos bioativos com propriedades antioxidantes, antimicrobianos, antidiabética e antiproliferativa/anticâncer, sendo usado como alimento funcional e aditivo alimentar (Smeriglio et al., 2016; Khoo et al., 2017).

Nas flores da CT foram identificados flavonoides como quercetina, campferol e glicosídeos de miricetina (Kazuma et al., 2003; Mukherjee et al., 2008). Entre as antocianinas, as ternatinas A1, A2, B1, B2, D1 e D2, de coloração azul, são as seis predominantes na CT (Chayaratanasin et al., 2015). Outros compostos lipofílicos foram identificados sendo ácidos graxos (palmítico, esteárico, petroselínicos, araquídico, beênico e fitânico), fitosterois (campesterol, estigmasterol, $\beta$-sitosterol e sitostanol) e tocois ( $\alpha$-tocopherol and -tocopherol) (Shen et al., 2016). Diante da significativa concentração de compostos bioativos, as flores de CT podem ser empregadas como fonte natural de antioxidantes e/ou como possível suplemento na indústria de alimentos e farmacêutica (Jeyaraj et al., 2021), visto que dependendo da concentração, os antioxidantes sintéticos podem apresentar toxicidade (Berton-Carabin, 2014).

Os métodos de extração de compostos fenólicos de plantas variam amplamente devido à natureza complexa da matriz e pela presença de flavonoides em diferentes formas. Além disso, o rendimento da extração dos compostos bioativos e a capacidade antioxidante são significativamente influenciados pelo tipo de solvente, devido às suas propriedades dielétricas e eficiência de extração variáveis, bem como pela variabilidade estrutural dos compostos almejados no processo. Portanto, a seleção adequada de solventes, a proporção ótima de sólido e líquido, o tempo e temperatura de extração são essenciais para 
alcançar alta eficiência (Banerjee \& Bonde, 2011; Mustafa \& Turner, 2011; Sheng et al., 2011; Nguyen et al., 2016).

Entre os solventes comumente utilizados na extração de fenólicos estão o etanol, metanol, acetona ou a mistura destes com água. Entretanto, para aplicação em alimentos, os fenólicos são preferencialmente extraídos com etanol por ser considerado de grau alimentar, biocompatível e mais econômico do que os outros solventes citados (Ilayaraja et al., 2015). Neste contexto, este estudo teve como objetivo avaliar o efeito da concentração de etanol na extração de compostos fenólicos da flor de Clitoria ternatea $\mathrm{L}$. e determinar a capacidade antioxidante.

\section{Material e Métodos}

O experimento foi realizado nos laboratórios de pesquisa do Programa de Pós-graduação em Tecnologia de Alimentos, da Universidade Tecnológica Federal do Paraná, campus Londrina. Trata-se de uma pesquisa aplicada, quantitativa, explicativa e estatístico (Pereira et al., 2018).

As flores de Clitoria ternatea L. foram coletadas em uma propriedade rural localizada na cidade de Nova América da Colina - PR, de fevereiro a abril de 2021. Para extração dos compostos fenólicos utilizou-se etanol absoluto (Alphatec, Brasil). Para análise de compostos fenólicos totais foram empregados reagentes de Folin-Ciocalteu (Sigma Aldrich, EUA), ácido gálico (Dinâmica, Brasil) e carbonato de sódio (Dinâmica, Brasil). Para o ensaio de capacidade antioxidante DPPH (2,2-difenil-1picril-hidrazil), Trolox (6-Hidroxi-2,5,7,8-tetrametilchroman-2-ácido carboxílico) e TPTZ (2,4,6-tri(2-piridil)-1,3,5-triazina) da Sigma Aldrich foram utilizados.

\subsection{Coleta e secagem das flores de Clitoria ternatea $\mathrm{L}$.}

As flores foram colhidas e mantidas em bandejas a temperatura ambiente por $48 \mathrm{~h}$ e em seguida foram secas em estufa com circulação forçada de ar a $50^{\circ} \mathrm{C}$ por 3 horas.

\subsection{Extração dos compostos fenólicos}

Em tubos Falcon pesou-se $1 \mathrm{~g}$ de amostra de flor seca e adicionou-se $25 \mathrm{~mL}$ de solvente. Diferentes concentrações de etanol em água $(0,20,40,60,80$ e $100 \%$, v/v) foram avaliadas. Os tubos foram agitados em agitador de tubos (Phoenix, Brasil) por 1 hora à temperatura ambiente, em seguida foram centrifugados e o sobrenadante coletado para determinação da concentração de compostos fenólicos totais e capacidade antioxidante. As condições de extração foram definidas por testes preliminares e a extração foi realizada em duplicata.

\subsection{Compostos fenólicos totais}

A concentração de compostos fenólicos totais foi determinada pelo método Folin-Ciocauteau (Singleton \& Rossi, 1965). Em tubo de ensaio adicionou-se $200 \mu \mathrm{L}$ de extrato, $1000 \mu \mathrm{L}$ da solução de Folin-Ciocalteu (10\%, v/v) e $800 \mu \mathrm{L}$ de solução de carbonato de cálcio (7,5\%, v/v). Um branco foi preparado com $200 \mu \mathrm{L}$ de água destilada, $1000 \mu \mathrm{L}$ da solução de Folin-Ciocalteu e $800 \mu \mathrm{L}$ de solução de carbonato de cálcio. Os tubos foram mantidos em ambiente escuro à temperatura ambiente por 30 minutos. A leitura da absorbância foi realizada a $750 \mathrm{~mm}$ em espectrômetro UV-VIS (Biochrom, modelo Libra, Inglaterra). Uma curva analítica de ácido gálico $\left(R^{2}=0,9944\right)$ foi utilizada para quantificação dos compostos fenólicos totais e foram expressos em mg de equivalente de ácido gálico por gramas de amostra (mg EAG/g de amostra).

\subsection{Capacidade antioxidante}

A capacidade antioxidante pelo método de redução do ferro (FRAP) foi de acordo com o método descrito por Rufino et al. (2006), com modificações. Em tubo de ensaio uma alíquota de $100 \mu \mathrm{L}$ do extrato foi misturado com $300 \mu \mathrm{L}$ de água 
destilada e $3000 \mu \mathrm{L}$ do reagente FRAP $(10 \mathrm{mM}$ TPTZ em $40 \mathrm{mM} \mathrm{HCl}$, mais $20 \mathrm{mM}$ cloreto férrico e $300 \mathrm{mM}$ de tampão acetato, $\mathrm{pH} 3,6,1: 1: 10 \mathrm{v} / \mathrm{v} / \mathrm{v})$. Os tubos foram agitados e mantidos em banho-maria a $37^{\circ} \mathrm{C}$ por 30 minutos para reação. A leitura foi realizada em espectrofotômetro UV-VIS (Biochrom, modelo Libra, Inglaterra) a $595 \mathrm{~nm}$ e o reagente FRAP empregado como branco para calibrar o equipamento. Uma curva analítica de Trolox $\left(R^{2}=0,9926\right)$ foi construída e os resultados expressos em mM Trolox equivalente por $\mathrm{g}$ amostra.

A capacidade antioxidante dos extratos pelo método de ensaio DPPH foi de acordo com Rufino et al. (2007). Em tubos de ensaio, uma alíquota de $100 \mu \mathrm{L}$ do extrato foi misturada com 3,9 mL de solução de DPPH 0,06 mM. Após 40 minutos a absorbância foi medida em espectrofotômetro (Biochrom, modelo Libra, Inglaterra) a $515 \mathrm{~nm}$. Os resultados foram calculados utilizando-se uma curva analítica de Trolox $\left(\mathrm{R}^{2}=0,9925\right)$ e expressos em $\mathrm{mM}$ de Trolox equivalente por $\mathrm{g}$ de amostra.

\subsection{Análise estatística}

Os resultados obtidos foram submetidos à análise de variância (ANOVA) e as diferenças estatísticas entre as médias identificadas pelo teste de comparação de médias de Tukey $(\mathrm{p}<0,05)$, utilizando-se o programa Statisticaß 12.0 (Statsoft, USA). O teste de correlação de Pearson foi realizado e os coeficientes de correlação (r) foram avaliados para determinar a relação entre os compostos fenólicos totais e capacidade antioxidante (DPPH e FRAP).

\section{Resultados e Discussão}

A concentração de compostos fenólicos dos extratos variou de 5,23 a 26,4 mg EAG/g amostra (Figura 1). Mehmood et al. (2019) obtiveram valores maiores de conteúdo de compostos fenólicos na extração convencional (72 mg EAG/g amostra) e por ultrassom ( $87 \mathrm{mg} \mathrm{EAG} / \mathrm{g}$ amostra) a $50^{\circ} \mathrm{C}$ utilizando água como solvente. Possivelmente, o aquecimento favoreceu a extração de fenólicos, pois eleva a energia interna das moléculas, aumentando a difusão e solubilidade dos compostos.

Figura 1. Efeito da concentração de etanol no teor de compostos fenólicos totais de extratos de CT

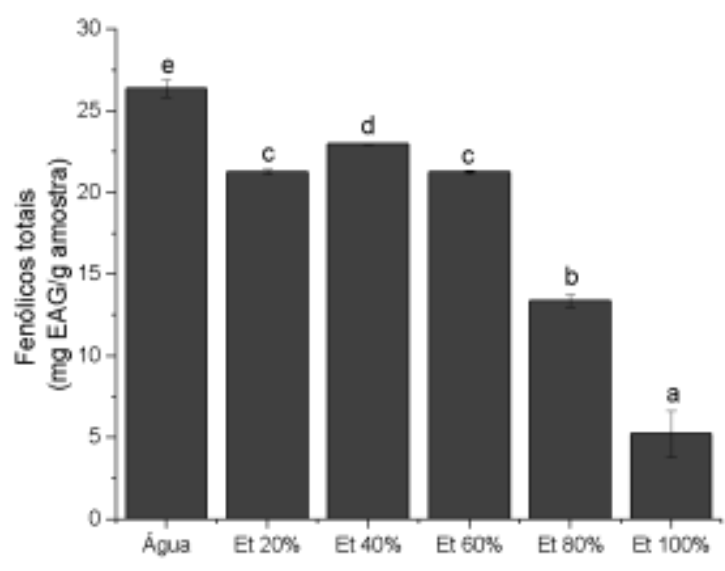

Fonte: Autores.

A concentração de etanol teve um efeito significativo $(\mathrm{p}<0,05)$ nos valores de compostos fenólicos dos extratos de CT, ou seja, conforme aumentou-se a concentração de etanol, diminuiu-se a quantidade de compostos fenólicos. Este comportamento pode ser atribuído ao aumento no inchamento da matriz da planta causada pela água, conduzindo à elevação da 
área de superfície de contato entre o soluto e solvente, melhorando o rendimento de extração de fenólicos (Kormin et al., 2010). Grande parte dos estudos associados à extração de compostos bioativos de CT relatam que o uso de solventes aquosos em mistura com metanol ou etanol proporcionam maiores rendimentos (Makasana et al., 2017). Porém, no presente estudo não foi observado o mesmo comportamento, podendo ser associado a diferentes temperaturas, tempo e proporção sólido e líquido de extração empregados. Em estudo similar, as condições ótimas de extração de CT foi a $54{ }^{\circ} \mathrm{C}, 74$ minutos, razão sólido e líquido de 1:37 e água como solvente (Baskaran et al., 2019), sugerindo novamente a necessidade do uso de temperatura acima de $50{ }^{\circ} \mathrm{C}$ para melhorar ao rendimento de extração.

O efeito da concentração de etanol na capacidade antioxidante dos extratos de CT foi avaliado pelo método de redução do ferro (FRAP) e captura do radical DPPH e os resultados estão na Figura 2.

Figura 2. Efeito da concentração de etanol na capacidade antioxidante (FRAP e DPPH) de extratos de CT.
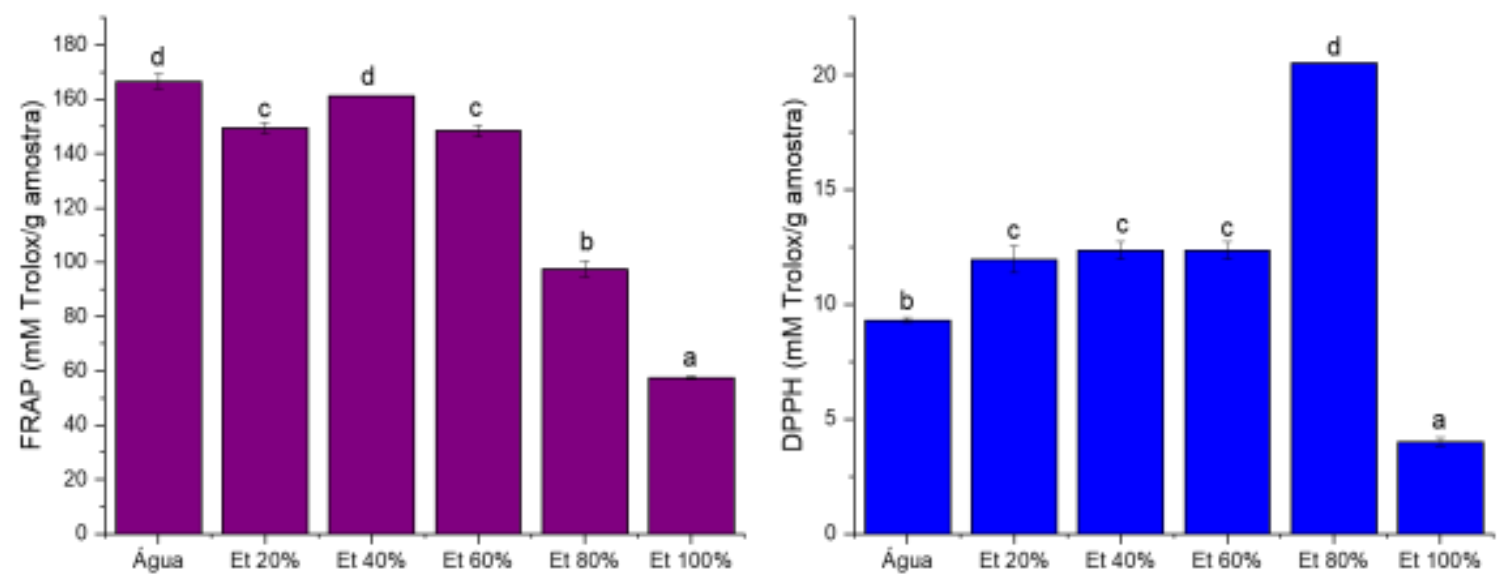

Fonte: Autores.

No ensaio FRAP houve redução na capacidade antioxidante conforme aumentou-se a concentração de etanol, correlacionando com os resultados de compostos fenólicos. Por outro lado, para o ensaio DPPH, houve incremento na capacidade antioxidante até $80 \%$ de etanol e com etanol puro o valor foi menor, igualmente ao ensaio FRAP. Os valores de capacidade antioxidante dos ensaios FRAP e DPPH foram bastante diversos e isso é em decorrência dos diferentes mecanismos de reação que estão envolvidos em cada método. O radical DPPH é colorido (violeta escuro) e quando em contato com um antioxidante, doa um átomo de hidrogênio e a forma reduzida do radical é gerada seguida pela perda de cor (Ali et al., 2008). Já o ensaio FRAP é caracterizado pela habilidade de transferir elétrons, que resulta na redução dos íons ferro na presença de compostos antioxidantes (Craft et al., 2012).

O uso de métodos com mecanismos de ação distintos é recomendado para uma ampla avaliação da atividade antioxidante de extratos fitoterápicos aquosos complexos (Avan et al., 2016). É também recomendado que ao avaliar a atividade antioxidante por diferentes métodos, a correlação entre estes e a composição fenólica sejam avaliadas. Assim, na Tabela 1 estão os coeficientes de correlação de Pearson, calculados para determinar a relação entre a concentração de compostos fenólicos totais e capacidade antioxidante (DPPH e FRAP) dos extratos de CT. De acordo com Taylor (1990), valores de correlação de Pearson $\leq 0.35$, representam correlação fraca, de 0.36 a 0.67 , correlação moderada e de 0.68 a 1.00 , forte correlação. Assim, pode-se afirmar que apenas os compostos fenólicos e FRAP apresentaram uma forte correlação (0.9928). 
Tabela 1. Correlação de Pearson entre fenólicos e capacidade antioxidante dos extratos de CT.

\begin{tabular}{lcc}
\hline & DPPH & FRAP \\
\hline Fenólicos & 0,2087 & 0,9928 \\
DPPH & 1 & 0,1893 \\
\hline
\end{tabular}

Fonte: Autores.

\section{Conclusão}

Neste estudo, a água em temperatura ambiente mostrou-se como melhor solvente para extração de compostos fenólicos das flores de Clitoria ternatea L. Entre os métodos de atividade antioxidante avaliados, apenas FRAP mostrou forte correlação com a concentração de compostos fenólicos totais.

Sugere-se a continuidade deste estudo empregando diferentes tempos e temperatura de extração, visando maximizar a concentração de compostos bioativos e avaliar a sua aplicabilidade em alimentos e bebidas. Destaca-se também a possibilidade de empregar tecnologias emergentes como ultrassom e aquecimento ôhmico para incrementar o rendimento de extração dos compostos bioativos de CT.

\section{Agradecimentos}

Os autores agradecem ao Conselho Nacional de Desenvolvimento Científico e Tecnológico (CNPq) pela bolsa e pelo auxílio financeiro e ao Laboratório Multiusuário da UTFPR - campus Londrina (Labmulti-LD).

\section{Referências}

Ali, S. S., Kasoju, N., Luthra, A., Singh, A., Sharanabasava, H., \& Sahu, A. (2008). Indian medicinal herbs as sources of antioxidants. Food Research International, 41, 1-15.

Avan, A. N., Demirci Çekiç, S., Uzunboy, S., \& Apak, R. (2016). Spectrophotometric determination of phenolic antioxidants in the presence of thiols and proteins. International Journal of Molecular Sciences, 17(8), 1325.

Banerjee, S. K., \& Bonde, C. G. (2011) Total phenolic content and antioxidant activity of extracts of Bridelia Retusa Spreng Bark: Impact of dielectric constant and geographical location. Journal of Medicinal Plants Research, 5(5), 817-822.

Baskaran, A., Mudalib, S. K. A., \& Izirwan, I. (2019). Optimization of aqueous extraction of blue dye from butterfly pea flower. Journal of Physics: Conference Series, 1358, 12001.

Berton-Carabin, C. C., Ropers, M. H., \& Genot, C. (2014). Lipid Oxidation in oil-in-water emulsions: Involvement of the interfacial layer. Comprehensive Reviews in Food Science and food Safety, 13(5), 945-977.

Boroski, M., Visentainer, J. V., Cottica, S. M., \& Morais, D.R. (2015). Antioxidantes: princípios e métodos analíticos, $1,141$.

Castañeda-Ovando, A., Pacheco-Hernández, M., Páez-Hernández, M., Rodríguez, J. A., \& Galán-Vidal, C. A. (2009). Chemical studies of anthocyanins: A review. Food Chemistry, 113, 859-871.

Chayaratanasin, P., Barbieri, M. A., Suanpairintr, N., \& Adisakwattana, S. (2015). Inhibitory effect of Clitoria ternatea flower petal extract on fructoseinduced protein glycation and oxidation-dependent damages to albumin in vitro. BMC Complementary and Alternative Medicine, 15(27), 1-9.

Craft, B. D., Kerrihard, A. L., Amarowicz, R., \& Pegg, R. B. (2012). Phenol-based antioxidants and the in vitro methods used for their assessment. Comprehensive Reviews in Food Science and Food Safety, 11, 148-173.

Ilayaraja, N., Likhith, K. R., Babu, G.R.S., \& Khanum, F. (2015). Optimisation of extraction of bioactive compounds from Feronia limonia (wood apple) fruit using response surface methodology (RSM). Food Chemistry, 173, 348-354.

Kormin, F., Ahmed, I., Yunus, R. M., \& Yusof, Z. A. M. (2010). Thepotential of modified microwave extraction system (MMES) to extract bioactive components from ferns. International Journal of Engineering and Technology, 10, 7-21.

Khoo, H. E., Azlan, A., Tang, S. T., \& Lim, S. M. (2017). Anthocyanidins and anthocyanins: Colored pigments as food, pharmaceutical ingredients, and the potential health benefits. Food \& Nutrition Research, 61(1), 1361779.

Makasana, J., Dholakiya, B. Z., Gajbhiye, N. A., \& Raju, S. (2017). Extractive determination of bioactive flavonoids from butterfly pea (Clitoria ternatea Linn.). Research on Chemical Intermediates, 43, 783-799. 
Research, Society and Development, v. 10, n. 11, e218101119450, 2021

(CC BY 4.0) | ISSN 2525-3409 | DOI: http://dx.doi.org/10.33448/rsd-v10i11.19450

Mehmood, A., Ishaq, M., Zhao, L., Yaqoob, S., Safdar, B., Nadeem, M., Munir, M., \& Wang, C. (2019). Impact of ultrasound and conventional extraction techniques on bioactive compounds and biological activities of blue butterfly pea flower (Clitoria ternatea L.). Ultrasonics sonochemistry, 51, $12-19$.

Morris, J. (2009). Characterization of butterfly pea (Clitoria ternatea L.) accessions for morphology, phenology, reproduction and potential nutraceutical, pharmaceutical trait utilization. Genetic Resources and Crop Evolution, 56, 421-427.

Mustafa, A., \& Turner, C. (2011). Pressurized liquid extraction as a green approach in food and herbal plants extraction: A review. Analytica Chimica Acta, $703,8-18$.

Nguyen, V. T., Bowyer, M. C., Vuong, Q. V., Altena, I. A. V., \& Scarlett, C. J. (2016). Phytochemicals and antioxidant capacity of Xao tam phan (Paramignya trimera) root as affected by various solvents extraction methods. Industrial Crops and Products, 67, 192-200.

Oliveira, H. A. B. (2019). Habits and food crops on the consumption of nonconventional vegetables by family farmers. Revista Agrária Acadêmica, 2(3), 1732.

Oliveira, H.A.B., Anunciação, P. C., Silva, B. P., Souza, A. M. N., Pinheiro, S. S., Lucia, C. M. D., Cardoso, L. M., Castrol, L. C. V., \& Podsędek, A. (2007). Natural antioxidants and antioxidant capacity of Brassica vegetables: A review. LWT-Food Science and Technology, 40 (1), 111.

Pereira, A. S., Shitsuka, D. M., Parreira, F. J., \& Shitsuka, R. (2018). Metodologia da Pesquisa Científica. Santa Maria: UAB/NTE/UFSM.

Rufino, M. S. M. (2006). Metodologia científica: Determinação da atividade antioxidante total em frutas pelo método de redução do ferro (FRAP). Embrapa, Comunicado técnico 125 .

Rufino, M. S. M. (2007). Metodologia científica: Determinação da atividade antioxidante total em frutas pela captura do radical livre DPPH. Embrapa, Comunicado técnico 127.

Shen, Y., Du, L., \& Zeng, H. (2016). Butterfly pea (Clitoria ternatea) seed and petal extracts decreased Hep-2 carcinoma cell viability. International Journal of Food Science and Technology, 51, 1860-1868.

Sheng, Z. L., Wan, P. F., Dong, C. L., \& Li, Y. H. (2013). Optimization of yotal flavonoids content extracted from Flos populi using Response Surface Methodology. Industrial Crops and Products, 43, 778-786.

Singleton, V. L., \& Rossi, J. A. (1965). Colorimetry of total phenolics whif phophomolybdic-phospotungstic acid reagentes. American Journal of Enology and Viticulture, 16, 144-158.

Smeriglio, A., Barreca, D., Bellocco, E., \& Trombetta, D. (2016). Chemistry, pharmacology and health benefits of anthocyanins. Phytotherapy Research: PTR, $30(8), 1265-1286$.

Taylor, R. (1990). Interpretation of the correlation coefficient: a basic review. Journal of. Diagnostic Medical Sonography, 6, 35-39.

Zingare, M. L., Zingare, P.L., Dubey, A. K., \& Ansari, M. A. (2013). Clitoria ternatea (APARAJITA): A review of the antioxidant, antidiabetic and hepatoprotective potential. International Journal of Pharmacy and Biological Science, 3(1), 201-210. 